DOI: $10.5433 / 2317-627 X .2017 v 5 n 2 p 1$

\title{
EXPEDIENTE
}

\section{E-ISSN 2317-627X}

\section{ECONOMIA \& REGIÃO}

\author{
V.5, N.2, Jul./Dez. 2017 \\ EDITOR CHEFE \\ Prof. ${ }^{\text {a }}$ Dra. Marcia Regina Gabardo da Camara
}

\section{CONSELHO EDITORIAL}

$\mathrm{Dr}^{\mathrm{a}}$. Augusta Pelinski - Universidade Estadual de Ponta Grossa - Brasil

$\mathrm{Dr}^{\mathrm{a}}$. Denise Barros de Azevedo, Universidade Federal de Mato Grosso do Sul - UFMS, Brasil

Dr. Edson Talamini - Universidade Federal do Rio Grande do Sul - UFRGS, Brasil

Dra. Erlaine Binotto - Universidade Federal da Grande Dourados - UFGD, Brasil

Dr. Gilberto Joaquim Fraga, Universidade Estadual de Maringá - UEM, Brasil

Dr. Jandir Ferrera de Lima - Universidade Estadual do Oeste do Paraná/Toledo - Brasil

Dr. João Carlos Lopes - Universidade Técnica de Lisboa- Portugal

Dr. Joaquim José Martins Guilhoto - OCDE - Portugal

Dr. José Luiz Parré - Universidade Estadual de Maringá - Brasil

$\mathrm{Dr}^{\mathrm{a}}$. Leonor Silva Carvalho - Universidade de Évora -Portugal

$\mathrm{Dr}^{\mathrm{a}}$. Letícia de Oliveira, Universidade Federal do Rio Grande do Sul - UFRGS, Brasil

Dra $^{\text {a }}$ Marcia Regina Gabardo da Camara - Universidade Estadual de Londrina - Brasil

Dr. Pery Francisco de Assis Shikida - Universidade Estadual do Oeste do Paraná - Brasil

$\mathrm{Dr}^{\mathrm{a}}$. Rossana Lott Rodrigues - Universidade Estadual de Londrina - Brasil

Dr. Umberto Antonio Sesso Filho - Universidade Estadual de Londrina - Brasil

Dr. Valdemiro Alcântara da Silva Neto - Universidade Federal de Goiás - UFG, Brasil

\section{CONSELHO CONSULTIVO}

Angélica Massuquetti - UNISINOS

Augusta Pelinski - UEPG

Carlos Eduardo Caldarelli - UEL

Carlos Roberto Ferreira - UEL

Cleise Maria de Almeida Tupich Hilgemberg - UEPG

Eduardo Luis Casarotto - UFGD

Eliane Cristina de Araújo Sbardellati -UEM

Fernanda Mendes Bezerra Baço - UNIOESTE/Francisco Beltrão

Fernando Motta Correia -UFPr

Gilberto Joaquim Braga - UEM

Jackelline Favro - UEM

Joanna Giorgios Alexopoulos -UEL

Irene Domenes Zapparoli -UEL

Kalinka Léia Becker - Universidade Federal de Santa Maria - UFSM

Katy Maia-UEL

Leandro Gilio, ESALQ/USP

Luiz Gustavo Antonio de Souza - UNICAMP

Marcia Regina Gabardo da Camara -UEL

Marcia Regina Godoy - UNISINOS

Marcio Marconato- UEM

Margarida Garcia de Figueiredo- Universidade Federal de Mato Grosso - UFMT

Maria de Fátima Sales- UEL

Maria Helena Ambrósio Dias -UEM

Marina da Silva Cunha - UEM

Mirian Beatriz Schneider -Unioeste

Ricardo Luis Lopes- UEM

Rossana Lott Rodrigues -UEL

Sérgio Carlos de Carvalho - UEL

Solange de Cássia Inforzato de Souza - UEL

Tiago Santos Telles - IAPAR

Vanderlei José Sereia -UEL

Vera Luci Almeida -_Universidades Federal de Grande Dourados- UFGD 


\section{EDITORIA TÉCNICA}

Elianara Gomes dos Santos

\section{CONTATO}

Contato da revista

Prof. ${ }^{a}$ Dra. Marcia Regina Gabardo da Camara - UEL

Economia \& Região

Universidade Estadual de Londrina

CESA-Departamento de Ciências Econômicas

Rodovia Celso Garcia Cid | Pr 445 Km 380

Campus Universitário Cx. Postal 10.011 | CEP 86.057-970 | Londrina - PR

Telefone: 5543 3371-4255

e-mail: rer@uel.br

Contato de suporte

laudi@uel.br

Telefone: 5543 3371-4209 